\title{
OURICURI
}

\section{PREVISÕES DA PRODUÇÃo AGROPECUÁRIA E REPRODUÇÃo DE Podocnemis expansa (SCHWEIGGER, 1812) NO ENTORNO DA ILHA DO BANANAL NO ESTADO DO TOCANTINS}

\author{
Vailton Alves de FARIA ${ }^{1}$; Adriana MALVÁSIO²; Clauber ROSANOVA ${ }^{3}$
}

\author{
1Doutorando - Programa de Pós-Graduação em Ciências do Ambiente, da Universidade Federal do \\ Tocantins, Av. NS 15 ALC NO 14, Bloco III, Sala 15-A, Palmas/TO; CEP \\ 77020-210 - Palmas, TO - Brasil. E-mail: vailton@uft.edu.br. \\ ${ }^{2}$ Fundação Universidade Federal do Tocantins/UFT, Campus Universitário de Palmas, Rua ALC NO14, s/n. \\ Av. NS 15, CEP 77020-210 - Palmas, TO - Brasil. E-mail: malvasio@uft.edu.br. \\ ${ }^{3}$ Instituto Federal de Educação, Ciência e Tecnologia do Tocantins - IFTO, Campus Palmas. \\ Av. NS 15, ALCNO, 14, 109 Norte, CP 114, CEP 77001-090, Palmas, TO, Brasil. 'Doutorando - Programa \\ de Pós-Graduação em Ciências do Ambiente, da Universidade Federal do Tocantins. E-mail: \\ clarberzootec@hotmail.com. \\ http://doi.org/10.29327/ouricuri.v8.i2.a6
}

Resumo: O estudo objetivou apresentar e analisar as projeções da produção agropecuária dos municípios de Caseara, Cristalândia, Formoso do Araguaia, Lagoa da Confusão, Marianópolis e Pium com a quantidade de fêmeas de quelônios da espécie $P$. expansa nidificando nas praias do rio Javaés durante o período de execução do Projeto Quelônios da Amazônia (PQA), compreendido entre 1985 e 2010. Os dados da produção agropecuária foram analisados para a série histórica de 1990 a 2015, através do número de cabeças de gado de corte e área plantada com lavoura temporária e lavoura permanente, para o conjunto dos municípios estudados. Para análise e previsão dos dados, utilizou-se o modelo de séries temporais autorregressivo integrado de médias móveis. A partir da análise dos dados da produção agropecuária, observa-se que, em 1990, havia 73,5\% da área coberta por formações savânicas (domínio de Cerrado), passando em 2015, para $62,1 \%$, uma queda de $11,4 \%$. Em relação a quantidade de fêmeas de $P$. expansa nidificantes, a série de dados se mostrou instável, com períodos curtos de crescimento e decrescimento não sendo possível estabelecer um padrão dessas variáveis com a produção agropecuária na região. De forma geral, os resultados mostram que há outras variáveis "aparentemente" causadoras de menor impacto ao meio ambiente, como as variações climáticas, que apresentam mais efeitos sobre a população de fêmeas nidificantes nas praias do rio Javaés do que a expansão agropecuária na região.

Palavras-chave: Agropecuária; Séries Históricas; Nidificação; Quelônios; Reprodução.

\section{PREDICTIONS OF AGRICULTURAL PRODUCTION AND REPRODUCTION OF Podocnemis expansa (Schweigger, 1812) IN THE ENVIRONMENT OF BANANAL ISLAND IN THE STATE OF TOCANTINS}

\begin{abstract}
The study aimed to present and analyze the projections of agricultural production in the municipalities of Caseara, Cristalândia, Formoso do Araguaia, Lagoa da Confusão, Marianópolis and Pium, with the number of females of $P$. expansa chelonians nesting on the beaches of the Javaés river during the period of the Project Chelonians of Amazônia (PQA) from 1985 to 2010. Data on agricultural production were analyzed for the historical series from 1990 to 2015, through the number of heads of cattle and area planted with temporary crops and permanent crops all for the set of municipalities studied. For the data analysis and prediction, we used the integrated
\end{abstract}


autorregressive time series model of moving averages. From the analysis of agricultural production data, in 1990 , there was $73.5 \%$ of the area covered by savanna formations (Cerrado domain), in 2015 , to $62.1 \%$, a fall of $11,4 \%$. In relation to the number of females of $P$. expansa nesting, the data series showed to be unstable, with short periods of growth and decrease, it being not possible to establish a pattern of these variables with the agricultural production in the region. In general, the results of the analysis show that there are other "apparently" variables that cause less impact to the environment, such as climatic variations, which have more effects on the nesting females population on the beaches of the Javaés river than the agricultural expansion in the region.

Keywords: Farming, Historical Series; Nesting; Chelonians; Reproduction.

\section{PREVISIONES DE LA PRODUCCIÓN AGROPECUARIA Y REPRODUCCIÓN DE Podocnemis expansa (Schweigger, 1812) EN EL ENTORNO DE LA ISLA DEL BANANAL EN EL ESTADO DE TOCANTINS}

Resumen: El estudio objetivó presentar y analizar las proyecciones de la producción agropecuaria de los municipios de Caseara, Cristalândia, Formoso del Araguaia, Lagoa da Confusión, Marianópolis y Pium con la cantidad de hembras de quelonios de la especie $P$. expansa nidificando en las playas del río Javaés durante el período de ejecución del Proyecto Quelonios de la Amazonía (PQA), comprendido entre 1985 y 2010. Los datos de la producción agropecuaria fueron analizados para la serie histórica de 1990 a 2015, a través del número de cabezas de ganado de corte y área plantada con labranza temporal y labranza permanente para todos los municipios estudiados. Para el análisis y previsión de los datos, se utilizó el modelo de series temporales autoregresivo integrado de medias móviles. A partir del análisis de los datos de la producción agropecuaria, se observa que, en 1990, había el 73,5\% del área cubierta por formaciones savánicas (dominio de Cerrado), pasando a 2015, al 62,1\%, una caída de 11, 4\%. En cuanto a la cantidad de hembras de $P$. expansa nidificantes, la serie de datos se mostró inestable, con períodos cortos de crecimiento y decrecimiento no siendo posible establecer un patrón de esas variables con la producción agropecuaria en la región. En general, los resultados del análisis muestran que hay otras variables "aparentemente" causantes de menor impacto al medio ambiente, como las variaciones climáticas, que presentan más efectos sobre la población de hembras nidificantes en las playas del río Javaés que la expansión agropecuaria en la región región.

Palabras Chave: Agropecuaria; Series Históricas; Nidificación; Tortugas; Reproducción.

\section{INTRODUÇÃO}

A região da "Amazônia Legal" apresenta uma grande diversidade biológica, sendo que nos últimos anos foi notória a busca no equilíbrio da fauna silvestre através de ações de manejo sustentável e preservação de ambientes de grande diversidade biológica, porém, mesmo diante dessas ações a estabilidade na fauna tem sido alterada de forma constante e essa alteração, além de coibir o desenvolvimento sustentável representa também a extinção de muitas espécies (MMA, 2016).

A preocupação em utilizar os recursos faunísticos garantindo sua existência para gerações futuras tem motivado o planejamento e desenvolvimento de metodologias que busquem assegurar a conservação dessa riqueza.

Revista Ouricuri, Juazeiro, Bahia, v.8, n.2. p.026-048. jul./dez., 2018.

http://www.revistas.uneb.br/index.php/ouricuri | ISSN 2317-0131 
O estado do Tocantins, embora pertença formalmente à região Norte, está totalmente inserido na "Amazônia Legal" e encontra-se na zona de transição geográfica entre o Cerrado e a Floresta Amazônica (IBGE, 2007). Dos cinco grandes tipos de vegetação que formam as províncias vegetacionais que cobrem o país, o Tocantins apresenta duas: a Floresta Amazônica de terra firme, e a Savana, denominados, respectivamente, como Biomas Amazônia e Cerrado. Além destas regiões, ocorrem as Áreas de Tensão Ecológica e as formações pioneiras. Estas últimas quase sempre relacionadas a ambientes aquáticos, possibilitando o desenvolvimento de várias espécies faunísticas como répteis e anfíbios (FELFILI et al., 2005).

A dinâmica de ocupação do solo em Tocantins se torna mais preocupante em áreas de grande importância para a biodiversidade e preservação de ecossistemas, nesse sentido a região da Ilha do Bananal se apresenta com grande destaque. Configura desde 1959 como reserva ambiental com área de cerca de 20 mil quilômetros quadrados. Subdividida em duas partes: Parque Nacional do Araguaia (PNA) ao norte, representando praticamente toda sua extensão, sua área também se encontra nos municípios de Pium, Lagoa da Confusão e Formoso do Araguaia. Ao sul, o Parque Indígena do Araguaia, criado em 1971, possui cerca de 1.600 hectares, onde vivem índios das tribos Javaes e Carajás. Sua flora é típica do Cerrado e da Floresta Amazônica (MMA, 2001).

Presentes no estado do Tocantins, as Unidades de Conservação (UCs) Área de Proteção Ambiental Ilha do Bananal/Cantão (APA), Parque Estadual do Cantão (PEC) e PNA, pertencem a região descrita como Corredor Ecológico Araguaia/Bananal (CEA/B) e estão sendo sistematicamente alteradas por atividades humanas. É grande a interferência na região sendo mais acentuada na APA, onde o relevo plano coberto em sua maior parte por vegetação de Cerrado, impulsiona o desenvolvimento da agricultura e agropecuária (MMA, 2016).

O avanço das atividades agrícolas sobre as áreas das UCs no estado do Tocantins pode ter várias consequências para a fauna local. Bour (2008), afirma que os quelônios são extremamente sensíveis às modificações ambientais, são os primeiros grupos de vertebrados a desaparecerem quando seu habitat é destruído. A Podocnemis expansa (Schweigger, 1812) é uma das espécies de quelônios em que a população de fêmeas pode estar sendo alterada em função dessas atividades.

A alteração e degradação de habitats por atividades antrópicas levam muitas populações de quelônios a sobreviverem com baixas densidades (MMA, 2016). Portanto, se torna indispensável a investigação das consequências da intervenção do homem nesse ambiente, a fim de se estabelecer perspectivas e metodologias para a sua conservação e prospecção das espécies (MARQUÉZ, 1995).

Os quelônios representam uma importante fonte de alimento para habitantes da região amazônica. Ao longo dos anos a espécie $P$. expansa por ser de maior porte e a mais abundante 
na região, serviu de alimento para comunidades indígenas, ficando até mantida em cativeiro para ser utilizada na época da cheia dos rios, período em que os peixes eram mais escassos (SMITH, 1979). Segundo Rebêlo e Pezzuti (2000), a espécie atualmente ainda é consumida por populações tradicionais da Amazônia em diferentes níveis de exploração, assumindo importante papel social, econômico e cultural nas suas áreas de ocorrência. Segundo Soini (1999), é necessário um conjunto de ações estratégicas de gestão e manejo para garantir a sobrevivência e continuidade das espécies de quelônios aquáticos.

A $P$. expansa é a maior espécie do gênero Podocnemis, chegando a pesar cerca de $65 \mathrm{~kg}$ e medindo até $90 \mathrm{~cm}$ de comprimento. Ocorre praticamente em toda bacia do Rio Amazonas, desde o leste dos Andes até a Bacia do Rio Orinoco (VOGT, 2008). Atualmente, seu estado de conservação se encontra em quase ameaça de extinção (ICMBIO, 2014) e para a região amazônica como um todo, categoriza-se como criticamente ameaçada (IUCN, 2016).

No período de seca dos rios, os animais migram à procura dos locais de nidificação (VOGT, 2008). O período da desova pode variar de acordo com a localidade e o ciclo de cheia e seca dos rios. Nos rios Araguaia e Javaés, a nidificação ocorre entre os meses de setembro e outubro (PÁDUA, 1981; ALHO e PÁDUA, 1982; VOGT, 2008).

A manutenção e conservação dos quelônios de forma geral requerem conhecimentos específicos sobre a sua biologia reprodutiva que pode ser estudada a partir do número de ovos por ninhada, número de ninhos, tempo de incubação, sucesso de eclosão, número de filhotes e biometria (MARCOVALDI et al., 2011). Investigar aspectos ecológicos como distribuição e o tamanho populacional da $P$. expansa é essencial para subsidiar estratégias de proteção e manejo (MARCOVALDI et al., 2011), fornecendo dados relevantes para auxiliar na identificação de novas áreas de nidificação (MARCOVALDI e MARCOVALDI, 1985; SPOTILA, 2004).

Nesse sentido, a área do Corredor Ecológico Araguaia Bananal (CEAB) é considerada de grande relevância para a conservação dessa espécie. Além da conservação dos ecossistemas envolvidos, tem como um de seus principais objetivos, contribuir para a implementação de um modelo de desenvolvimento sustentável na região. Nessa área, são desenvolvidas ações de conservação, como o Quelônios da Amazônia (PQA), criado em 1990, pelo Centro Nacional de Quelônios da Amazônia (CENAQUA) através da Portaria IBAMA nำ 870/90, que em 2001, tornouse o Centro Nacional de Pesquisa e Conservação de Répteis e Anfíbios (RAN), que coordena e autoriza as atividades de manejo e conservação dos répteis e anfíbios brasileiros (IBAMA, 2004).

A fim de ressaltar a relevância da agropecuária e seus impactos causados na população de fêmeas de quelônios da espécie $P$. expansa, o presente estudo teve como objetivo apresentar e analisar projeções de produção agropecuária dos municípios de Caseara, Cristalândia, Formoso do Araguaia, Lagoa da Confusão, Marianópolis e Pium com a reprodução desses animais nas praias do rio Javaés. 
Os modelos usados para a obtenção das previsões se baseiam em modelos de séries temporais autorregressivos integrados de médias móveis (BOX e JENKINS, 1976). Os dados foram coletados para o período de 1990 a 2015 para produção agropecuária e de 1985 a 2010 para a reprodução da $P$. expansa.

\section{Modelos de Previsão para Séries Temporais}

Uma série temporal é denominada como sendo uma classe de fenômenos no qual o processo observacional e decorrente quantificação numérica gera uma sequência de dados distribuídos ao longo do tempo (MORETTIN e TOLOI, 1985). O principal objetivo da análise de uma série temporal é explicar o comportamento passado da série, visando a previsão e compreensão do seu comportamento futuro, buscando previsões de movimentos futuros e/ou de tendência.

Para Morettin e Toloi (1985), os principais métodos de decomposição garantem que uma série temporal é formada por um conjunto de componentes não-observáveis. Os modelos clássicos consideram a série composta por quatro elementos principais: variações cíclicas, irregulares e sazonais e variações de tendência (BOX et al., 1994).

\section{Variações Cíclicas, Irregulares e Sazonais}

As variações cíclicas apresentam um movimento oscilatório de longa duração que mostra a influência de fatores aleatórios de ação constante, indicando as fases de expansão e contração da variável em estudo para intervalos de duração variáveis (MORETTIN e TOLOI, 2006).

Morettin e Toloi (2006), explicam que, pelo fato de as variações cíclicas não apresentarem duração constante, a identificação da componente irregular se torna mais difícil. Tais variações não podem ser separadas de variações irregulares, levando a uma análise conjunta.

A componente sazonal representa as variações da série de acordo com algum fator de sazonalidade (MORETTIN e TOLOI, 2006). Ou seja, é um movimento oscilatório de curta duração que representam a influência de fatores com ação periódica aumentando ou diminuindo a intensidade do fenômeno.

\section{Variações de Tendência}

O aumento ou declínio gradual observado nos valores de uma série temporal é definido como tendência (WHEELWRIGHT, 1985). Determinar e identificar a tendência em uma série temporal permite estabelecer previsões e conhecer o comportamento de outras componentes da série, é obtido através do uso da análise de regressão por meio de uma função que indique o melhor comportamento da série. 


\section{Modelo ARIMA}

Os modelos ARIMA - Autorregressivos Integrados de Médias Móveis, formulados inicialmente na década de 70, por BOX et al. (1994), são modelos matemáticos que visam compreender o comportamento da autocorrelação entre os valores da série temporal, e com base nesse comportamento realizar previsões futuras.

Segundo o modelo, as variáveis apresentadas pelos valores na série temporal, são extremamente dependentes entre si, ou seja, cada valor pode ser explicado por valores prévios da série. Para Pellegrini e Fogliatto (2000), os modelos ARIMA representam a classe mais geral dos modelos usados para análise de séries temporais podendo ser usados de maneira satisfatória em séries estacionárias e não estacionárias.

Uma série é estacionária quando os dados oscilam em torno de um ponto de equilíbrio (FAVA, 2000). Ocorre quando as propriedades da variável não são alteradas ao longo do tempo. Dentre os modelos aplicados a séries estacionárias tem-se: i) Modelo Auto Regressivo (AR), nesse caso a série é descrita por seus valores anteriores e um erro aleatório; ii) Modelo de Médias Móveis (MA), nessa modelagem a série resulta da combinação dos erros de previsão dos períodos passados com o atual e; iii) Modelo ARMA - Autorregressivo de Médias Móveis, que representa uma combinação dos dois anteriores.

Werner e Ribeiro (2003), afirmam que se variância e a média forem dependentes do tempo a série temporal é não estacionária. Box e Jenkins (1976), propuseram os modelos ARIMA que se baseiam na hipótese de que uma série temporal não estacionária pode ser tratada como uma série estacionária a partir de diferenciações e da inclusão de um componente $A R$ e um componente MA.

\section{Modelos Autorregressivo - AR}

Este modelo corresponde a um filtro, tendo como entrada o ruído branco da média e o desvio padrão e como saída o sinal desejado. Para Pellegrini e Fogliatto (2000) o valor atual do processo é uma combinação linear finita de valores prévios e um ruído aleatório $a_{t}$.

Em um modelo $A R$, a série de dados $Z_{t}$ é determinada por seus valores passados $Z_{t-1}, Z_{t-2}, \ldots, Z_{t-p}$ e pelo ruído branco. A estrutura autoregressiva total é apresentada na equação 01:

$$
\tilde{Z}_{t}=\phi_{1} \tilde{Z}_{t-1}+\phi_{2} \tilde{Z}_{t-2}+\ldots+\phi_{p} \tilde{Z}_{t-p}+a_{t}
$$


Onde os coeficientes $\phi_{1}, \phi_{2}, \ldots, \phi_{p}$ descrevem como os valores de $Z_{t}$ relacionam-se com as variáveis passadas $Z_{t-1}, Z_{t-2}, \ldots, Z_{t-p}$.

\section{Modelo de Médias Móveis - MA}

Os modelos de MA são criados a partir da combinação linear do ruído aleatório, $a_{t}$, ocorrido no período atual e no período passado. Nesse modelo, $\tilde{Z}_{t}$, que representa $Z_{t}$ subtraída da média $\mu$, depende linearmente de um número finito $q$ de valores prévios do ruído aleatório $a_{t}$. Para Pellegrini e Fogliatto (2000), um processo aleatório de ordem $k$ é dado pela equação 02 :

$$
Z_{t}=a_{t}-\theta_{1} a_{t-1}-\theta_{2} a_{t-2}-\ldots-\theta_{q} a_{t-q} \quad 02
$$

Onde os coeficientes $\theta_{1}, \theta_{2}, \ldots, \theta_{q}$ são parâmetros da estrutura de $Z_{t}$.

\section{Modelo AR e MA - ARMA}

Neste modelo, tem-se uma combinação dos modelos AR e MA, no qual $Z_{t}$ é obtido por seus valores passados e pelos ruídos aleatórios $a_{t}$ atual e passado. Box et al. (1994), afirmam que algumas séries são melhor modeladas com a inclusão de termos AR e MA, o resultado é um modelo misto ARMA de ordem $(p, q)$ como apresentado na equação 03:

$$
\tilde{Z}_{t}=\phi_{1} \tilde{Z}_{t-1}+\phi_{2} \tilde{Z}_{t-2}+\ldots+\phi_{p} \tilde{Z}_{t-p}+a_{t}-\theta_{1} a_{t-1}-\theta_{2} a_{t-2}-\ldots-\theta_{q} a_{t-q}
$$

O modelo possui $p+q+2$ parâmetros desconhecidos, que podem ser estimados a partir dos valores observados na série temporal. Normalmente, os valores de $p$ e $q$ são menores que 2 para séries estacionárias (BOX et al., 1994).

\section{Modelagem ARIMA}

A análise e previsão de séries temporais nos quais os processos estocásticos não são estacionários podem ser realizados de forma satisfatória através da aplicação dos modelos ARIMA (BOX et al., 1994). Morettin et al. (2006), afirmam que nesse caso, a série original deverá ser diferenciada a fim de torná-la estacionária.

A associação de três componentes denominadas "filtros" representa a classe desse modelo, são eles: o componente autorregressivo (AR), o filtro de integração (I) e o componente de 
médias móveis (MA). O número necessário de diferenciação para tornar a série não estacionária em estacionária é chamado de ordem de integração (d) (WERNER e RIBEIRO, 2003).

Morettin e Toloi (2006), afirmam ser suficiente no máximo duas diferenças para que a série se torne estacionária. A inclusão do termo, ordem de integração $(d)$, permite que sejam utilizados modelos ARIMA $(p, d, q)$ dados pela equação 04 .

$$
w^{d}=\phi_{1} w_{t-1}+\ldots+\phi_{p} w_{t-p}+\varepsilon_{1}-\theta_{1} \varepsilon_{t-1}-\ldots-\theta_{q} \varepsilon_{t-q}
$$

Onde: $w_{t}=\Delta^{d} Z_{t}$, os coeficientes $\phi$ representam os operadores auto regressivos de ordem $p$ e $\theta$ os operadores de médias móveis de ordem $q$.

\section{Estacionariedade da Série}

A aplicação do modelo ARIMA exige que a série seja estacionária (BOX et al., 1994). Nesse caso, deve-se haver um desenvolvimento em torno de uma média, variância e autocovariância constantes na série, caso isso não ocorra é necessário a transformação da série de forma que ela se torne estacionária. Existem vários testes que podem ser usados para verificação da estacionariedade, que são denominados testes de raiz unitária, dentre eles: Augmented Dickey-Fuller (ADF), KPSS, Ljung-Box, Phillips-Perron, entre outros (MORETTIN e TOLOI, 1985).

Neste estudo optou-se pelo teste ADF (DICKEY e FULLER, 1979), eliminando assim o problema de autocorrelação dos resíduos através da incorporação de defasagens na expressão do teste. Essas defasagens são indicadas pelos critérios estatísticos Akaike Information Criterion (AIC) e Schwarz Bayesian Criterion (SBC). Nesse caso, baseou-se no critério (AIC) (ARÊDES e PEREIRA, 2008).

A equação do teste de raiz unitária ADF, com o componente constante é representado pela equação 05:

$$
\Delta Y_{t}=\beta_{1}+\delta Y_{t-1}+\alpha_{i} \sum_{i=1}^{m} \Delta Y_{t=i}+\varepsilon_{t}
$$

sendo $\Delta$ o operador de diferença de $Y_{t} ; \beta_{1}$ é o parâmetro constante, $\delta$ é o parâmetro da variável defasada e $\alpha_{\alpha_{i}} \sum_{i=1}^{m} \Delta Y_{t-i}$ é o termo de diferenças defasadas (ARÊDES e PEREIRA, 2008). 


\section{Critérios de Avaliação do Modelo}

Baseado em um ciclo interativo, a construção do modelo proposto $\operatorname{ARIMA}(p, d, q)$ é feita com referência aos próprios dados da série (MORETTIN e TOLOI, 1985). Segundo Box e Jenkins (1976), são necessárias três etapas para a criação do modelo: Identificação, estimação e verificação.

1. Identificação: Consiste em descobrir qual a melhor versão do modelo ARIMA deverá ser usado para descrever o comportamento da série, sendo esta sanonal ou não. A identificação do modelo se dá pelo comportamento das funções de autocorrelação (FAC) e das funções de autocorrelação parciais (FACP). Makridakis et al. (1998), apresentam mais detalhes referentes à obtenção das funções FAC e FACP e quais os comportamentos que representam o modelo abordado.

2. Estimação: É estabelecer os parâmetros do componente autorregressivo (AR), os parâmetros do componente de médias móveis (MA) e a variância do erro aleatório.

3. Verificação: Consiste em analisar se o modelo estimado é adequado para descrever o comportamento dos dados.

Caso o modelo não seja adequado, repete-se o ciclo, retornando a fase de identificação, caso seja satisfatório, passa-se para a última etapa, no caso, realizar as previsões da série.

Para Granger e Newbold (1986), a metodologia Box e Jenkins (1976) deve ser aplicada em séries temporais com mais de vinte observações, onde as características estruturais são desconhecidas. Fischer (1982), ressalta outro importante aspecto dos modelos ARIMA, relacionado ao limite do cenário de previsão, no qual, os modelos apresentam a característica geral de suas previsões em reverterem à média quando o cenário de previsão aumenta. Nesse sentido, o potencial de previsão destes modelos diminui a medida que o horizonte de previsão aumenta.

\section{MATERIAL E MÉTODOS}

\section{Área de estudo}

O estudo foi desenvolvido na região central do CEAB, área que abrange as UCs PNA, PEC, APA ilha do Bananal/Cantão e as áreas de abrangência dos municípios de Caseara, Cristalândia, Formoso do Araguaia, Lagoa da Confusão, Marianópolis e Pium (Figura 1). 


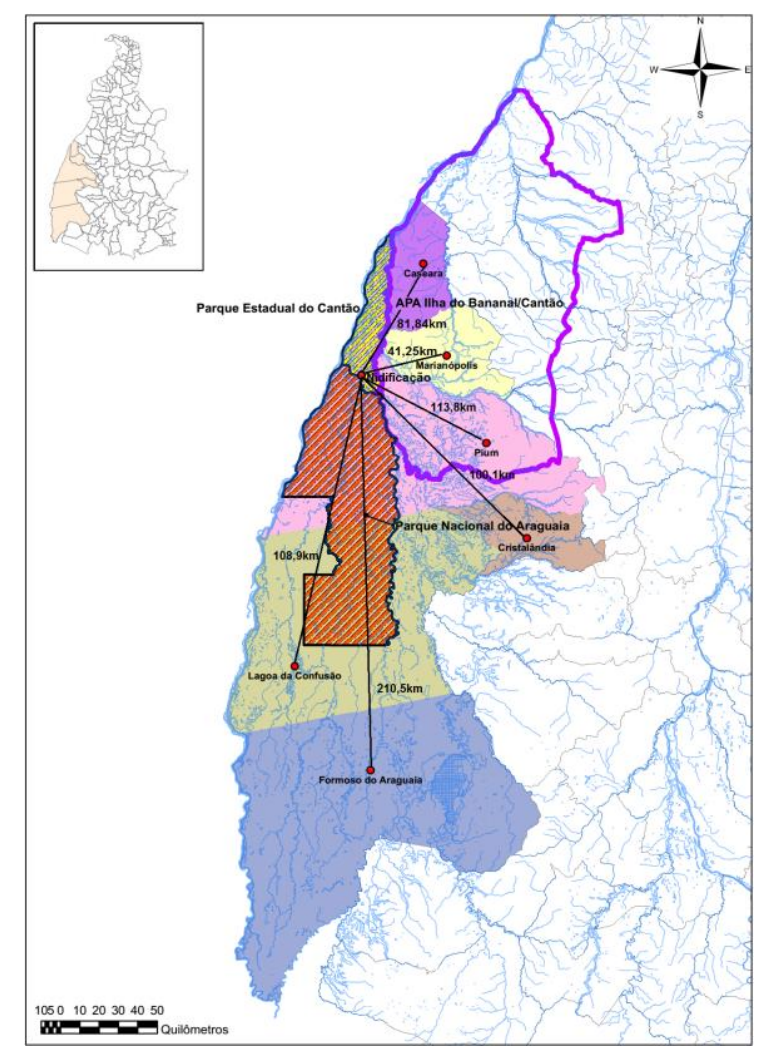

Figura 1. Localização do Parque Estadual do Cantão (PEC), Parque Nacional do Araguaia (PNA) e dos limites da Área de Proteção Ambiental Bananal Cantão (APA) Ilha do Bananal/Cantão e a área dos municípios onde se realizou o estudo com destaque para as distâncias em linha reta entre os municípios e o principal ponto de nidificação da Podocnemis expansa. Fonte: Os autores.

\section{Coleta de dados}

Os dados da produção agropecuária foram obtidos através de levantamento censitário no Instituto Brasileiro de Geografia e Estatística (IBGE), Empresa Brasileira de Pesquisa Agropecuária (EMBRAPA), Ministério do Meio Ambiente (MMA) e Instituto Brasileiro do Meio Ambiente e dos Recursos Naturais Renováveis (IBAMA).

A quantidade de ninhos de $P$. expansa marcados nas praias do rio Javaés foram obtidos através do banco de dados do grupo de pesquisa Crocodilianos e Quelônios da Região Norte (CROQUE) da Universidade Federal do Tocantins, PQA, Instituto Chico Mendes de Conservação da Biodiversidade (ICMBio) e Centro Nacional de Pesquisa e Conservação de Répteis e Anfíbios (RAN).

As observações e as previsões acerca da expansão da agropecuária sobre a região em estudo foram analisados para as variáveis: número de hectares de área plantada com Lavoura Temporária (LT) e Lavoura Permanente (LP) e o quantitativo de cabeças de gado de corte, todas para o conjunto dos municípios em estudo. Essas informações são referentes ao período de 1990 a 2015, totalizando 26 observações. 
Para a variável, número de ninhos de quelônios da espécie $P$. expansa, o período de análise correspondeu a 1985 - 2010 (Base de dados do PQA). Para a estimativa do número de animais considerou-se que cada ninho marcado correspondesse a uma fêmea em fase de reprodução (MARCO et al., 2011).

\section{Análise dos dados}

A metodologia utilizada para análise dos dados fundamenta-se na implantação de modelos ARIMA - Autoregressive Integrated Moving Average.

Para a concepção do modelo, através da metodologia Box e Jenkins (1976), seguiu-se o ciclo iterativo que iniciou com identificação do modelo, passando para a estimação dos parâmetros, logo em seguida a verificação, sendo o modelo adequado, realizou-se as previsões, caso contrário, retorna-se à estimação dos parâmetros e realiza-se novamente o ciclo.

\section{Identificação do Modelo}

A partir dos dados das séries temporais das variáveis em estudo, verificou-se a estacionariedade das mesmas através do teste de raiz unitária ADF, em casos onde a série era não-estacionária, foram realizadas diferenças sucessivas na série original até que a mesma se tornasse estacionária.

O próximo passo foi analisar as funções de autocorrelações (FAC) e de autocorrelações parciais (FACP), que indicaram os valores $p$ e $q$ do modelo $\operatorname{ARIMA}(p, d, q)$. O comportamento dessas funções indica qual o modelo a ser utilizado, bem como auxilia no uso dos testes de raízes unitárias para confirmar a estacionariedade da série. As funções FAC e FACP indicaram a ordem dos modelos autorregressivos e de média móvel, contudo, foi necessário verificar a estacionariedade da série após cada diferenciação.

Assim, em virtude da evidência de não estacionariedade da série, tomou-se a diferença da série original. Utilizando a primeira diferença e reaplicando o teste ADF, até obter a estacionariedade da série. Nesse caso, as indicações da ordem de integração do modelo, ou seja, $d=1$ para primeira diferenciação e $d=2$ para segunda diferenciação, quando necessário.

\section{Estimativa do Modelo}

Uma vez identificado os valores $(p, d, q)$ do modelo ARIMA, foram estimados os parâmetros do modelo proposto, da variância do resíduo e da constante, todos a $5 \%$ de significância $p<0,05(p-$ valor $)$. O processo de estimação requereu um método iterativo de cálculo. Inicialmente, identificou-se os componentes do modelo através de testes de raiz unitária e análise do correlograma. 
Após a estimação, procedeu-se com a verificação do modelo. Esta etapa foi realizada através da análise do comportamento dos resíduos. Quando estes apresentaram um processo tipo ruído branco, no qual a média é próxima de zero a variância é constante e as correlações não são significativas, a série foi tida como estacionária (ABRAHAM e LEDOLTER, 1983).

\section{Verificação do Modelo}

Nessa etapa, de posse do modelo identificado e estimado foi verificado se o mesmo era ou não adequado. Em caso positivo, ele foi utilizado para fazer previsões. Em caso negativo, foi identificado outro modelo e repetiu-se as etapas de estimativa e verificação. Para Fava (2000), as formas de verificação mais utilizadas são: análise de resíduos e avaliação da ordem do modelo, neste estudo optou-se pela primeira.

\section{Previsão do Modelo}

Após a realização dos três passos: identificação, estimação e verificação, foram calculadas as previsões para os valores futuros das séries em estudo. Para estimação dos testes e dos modelos supracitados, utilizou-se o software econométrico livre GRETL versão 1.9.14. Em todas as séries temporais a hipótese de não estacionariedade foi aceita em nível de 95\% de significância, ou seja, $p<0,05$ ( $p$-valor).

\section{RESULTADOS E DISCUSSÃO}

Procurando descrever os impactos das atividades agropecuárias na região de estudo, e a partir deles dimensionar uma relação entre a produção agropecuária e a população de fêmeas de $P$. expansa em fase de reprodução, foram priorizados os dados relativos à extensão de terras utilizadas nas atividades produtivas em lavoura temporária (LT), lavoura permanente (LP) e rebanho bovino de corte.

No que tange a produção agropecuária sobre as áreas de preservação ambiental no estado do Tocantins, pode se observar que a área plantada com LP apresenta significativa redução se comparados os períodos entre 1990 e 2015 (Figura 2). Observa-se um coeficiente de variação relativamente alto para LP, acima de 50\%, o que mostra uma grande variabilidade dos dados dessa série, mostrando que a média da série não é representativa (Tabela 1).

Dentre as séries analisadas, foi na produção de bovinos onde se tem uma maior homogeneidade dos dados, pois apresenta o menor coeficiente de variação $(16,8 \%)$ (Tabela 1). Já para a série de ninhos e LT, tem-se uma variação moderada dos dados, em torno de $40 \%$. Para Freund e Simon (2000), o coeficiente de variação expressa o quanto da escala de medida, representada pela média, é ocupada pelo desvio-padrão. 
Tabela 1. Análise descritiva das séries temporais, produção agropecuária e ninhos de quelônios.

\begin{tabular}{ccccc}
\hline Medidas de Tendência & LP & LT & Ninhos & Bovinos \\
\hline Média & 962,8 & 92.617 & 357,6 & 594.790 \\
Mediana & 815 & 82.987 & 334 & 616.720 \\
Máximo & 1815 & 198.110 & 660 & 737.560 \\
Mínimo & 51 & 47.294 & 109 & 407.490 \\
Desvio Padrão & 554,3 & 37.866 & 154,9 & 99.810 \\
Coeficiente de Variação (\%) & 57,6 & 40,8 & 43,3 & 16,8 \\
Total de Observações & 26 & 26 & 25 & 26 \\
\hline Lavoura permanente (LP); Lavoura & Temporária (LT). Fonte dos dados: IBGE/SIDRA, 2017; \\
IBAMA/PQA, 2016. & &
\end{tabular}

As observações e as previsões da produção agropecuária e reprodução de quelônios da espécie $P$. expansa, com seus respectivos intervalos de confiança ao nível de 95\%, são apresentados nas figuras 2 a 5 .

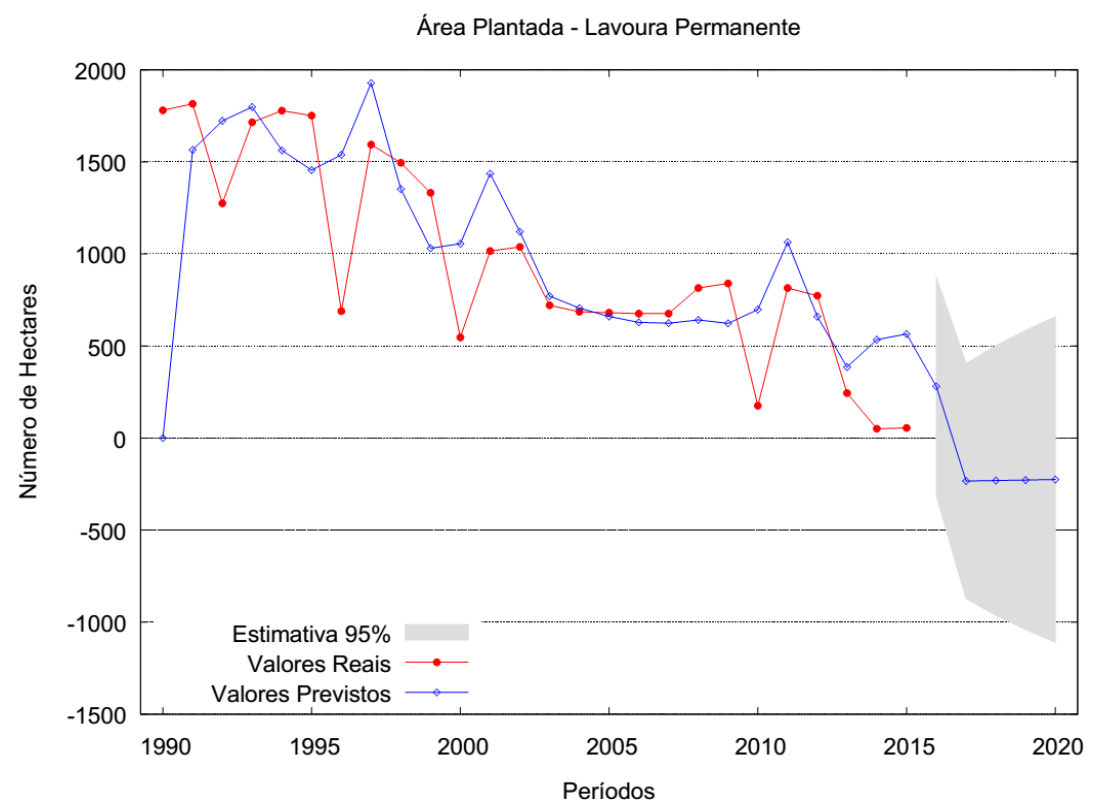

Figura 2. Projeção para área plantada com lavoura permanente para modelo ARIMA $(2,1,2)$ de 1990 a 2015 com previsão de cinco anos.

A LP, no contexto do uso da terra, é considerada toda a exploração com espécies de frutíferas como laranjeiras, cajueiros, coqueiros, bananeiras, cacaueiros em sistemas que combinam ou não culturas agrícolas e vegetação natural. Nesse sentido, a região apresenta uma redução significativa nas áreas destinadas a esse cultivo, como observado na Figura 2. 
Apresentando uma grande área de abrangência nos municípios, as áreas mapeadas como LT, caracterizam-se por culturas anuais de ciclo curto como soja, milho, algodão e feijão, tais culturas quase que triplicaram a área plantada entre 1990 e 2015, passando de aproximadamente 62.000 hectares em 1990 para mais de 190.000 hectares em 2015, ou seja, numa ascensão contínua desde 1990, o que confirma a previsão dada pelo modelo ARIMA $(2,1,2)$ (Figura 3).

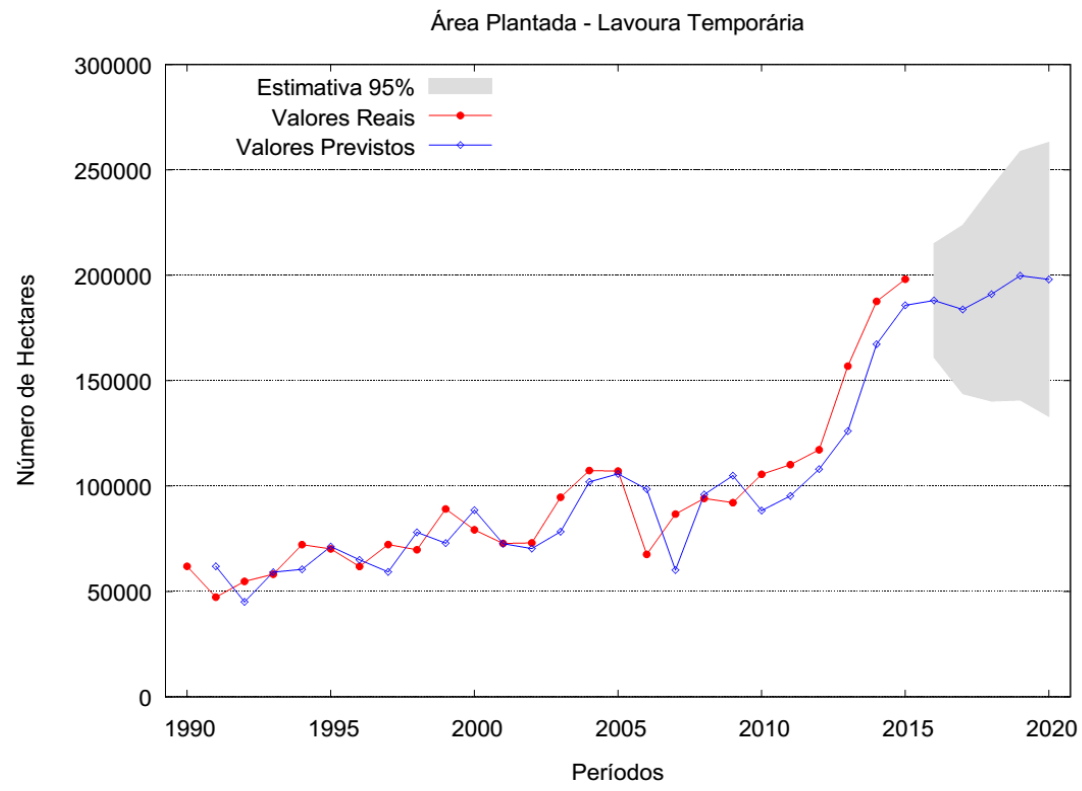

Figura 3. Projeção para número de hectares, área plantada com lavoura temporária, para modelo ARIMA $(2,1,2)$ de 1990 a 2015 com previsão de cinco anos.

De 1990 até 2015, a maioria das séries analisadas apresenta crescimento positivo, com exceção de LP, que teve seu pico máximo em 1990, com 1.780 ha plantados no conjunto dos municípios, caindo para 55 ha em 2015, com previsão para menos de 10 ha em 2020 . Por outro lado, as áreas plantadas com LT tiveram uma expansão significativa com tendência de crescimento no horizonte de cinco anos (Figura 3).

O município da Lagoa da Confusão, no ano de 1993 totalizava 11.104 hectares plantados, passando para 98.624 em 2015, apresentando um crescimento anual de 10,4\%. Em 1990, o município com maior percentual de terras destinadas a lavoura temporária era Formoso do Araguaia que respondia por $56,8 \%$, seguido por Cristalândia com $23,7 \%$ da área plantada. Em 1993, pela Lei Estadual № 498, de 21/12/1992, o distrito de Lagoa da Confusão é desmembrado do município de Cristalândia passando para segundo maior produtor de grãos entre os municípios analisados.

$\mathrm{Na}$ figura 4 são apresentadas as projeções do rebanho bovino, com base na série observada. O modelo ARIMA $(2,0,4)$ foi utilizado para mostrar o comportamento da produção, apresentando dois períodos curtos de queda ao longo do período projetado. 


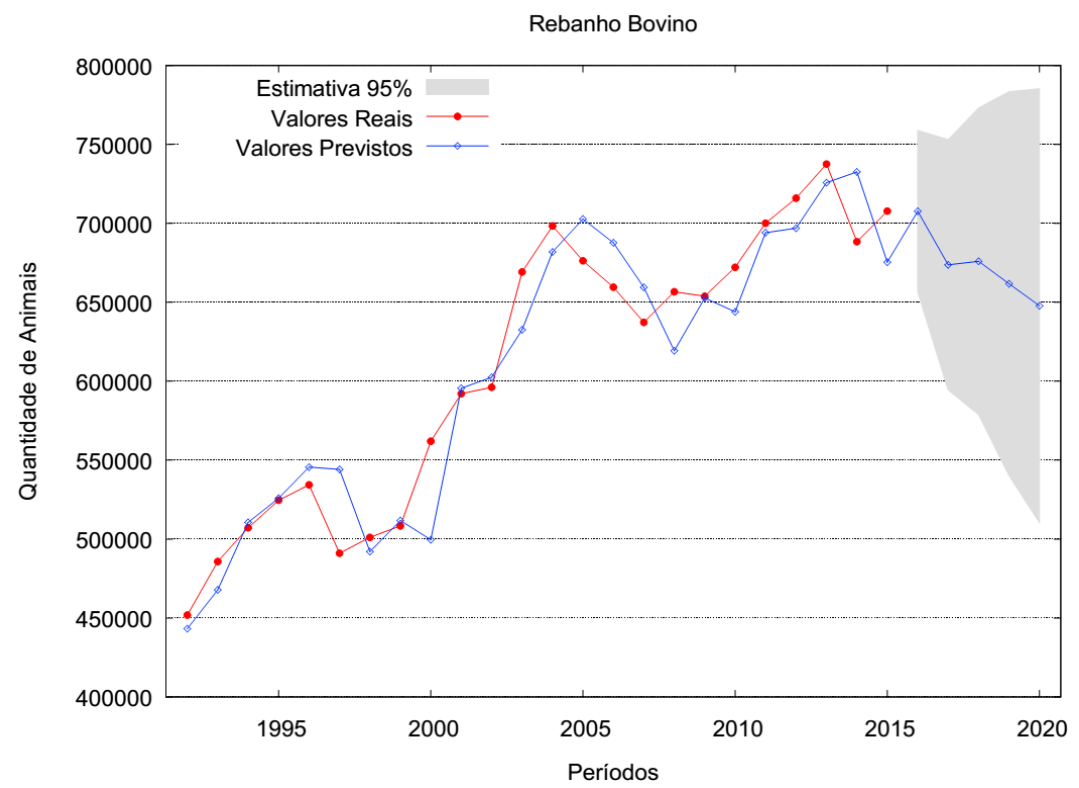

Figura 4. Projeção para quantidade do rebanho bovino de gado de corte, modelo ARIMA $(2,0,4)$ de 1990 a 2015 com previsão de cinco anos.

Em 1990, a pecuária era a atividade de maior relevância no estado do Tocantins. Nas microrregiões em estudo, em 1990, a densidade de bovinos era de 0,10 cabeças/hectare, em 2015, 25 anos depois, passa a ser de 0,17 animais por hectare, ou seja, um crescimento médio anual de 2,2\%. Um avanço significativo e preocupante frente aos danos que a bovinocultura representa para a cobertura florestal, integridade dos solos e impactos sobre os recursos hídricos, e consequentemente sobre a fauna local.

Segundo o IBGE, os rebanhos bovinos são os que apresentam números mais significativos em relação a ação antrópica sobre áreas de preservação ambiental. Em pesquisa realizada pela EMBRAPA Gado de Corte, em 2005, a bovinocultura de corte e leite no Brasil envolviam, no ano do estudo, 225 milhões de hectares para a criação de 195,5 milhões em todo o Brasil. A média nacional, de acordo com esses dados, seria de 0,88 cabeça por hectare. Essa média, certamente não é válida para região em estudo, mas permite afirmar que a bovinocultura ocupa na região algo superior a 3,9 milhões de hectares, esse crescimento pode ser confirmado através dos resultados apresentados pelo modelo proposto para essa série.

$\mathrm{Na}$ figura 5 são apresentados os valores observados na série temporal de 1985 a 2010 e as projeções para o número de ninhos de $P$. expansa em um horizonte de cinco anos, para os quais foi utilizado o modelo ARIMA $(2,1,2)$. Nota-se um modelo significativo, com $p<0,05$, entre a observação e a projeção da série a qual se mostrou praticamente constante para o horizonte previsto. 


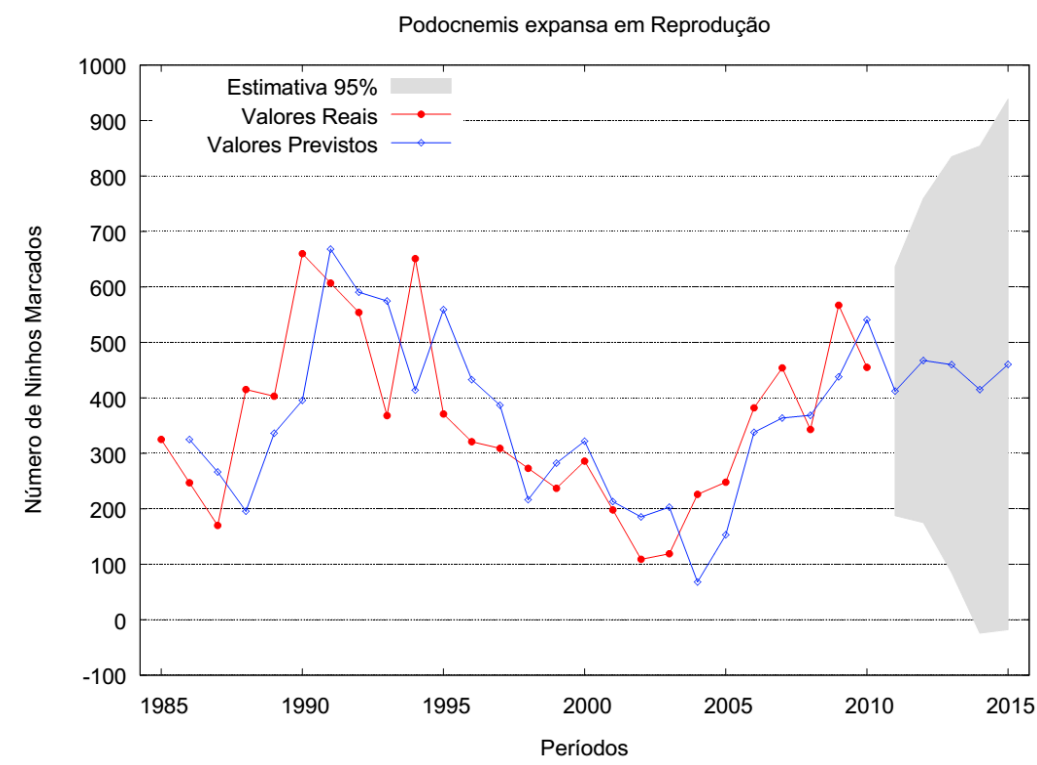

Figura 5. Projeção para reprodução de Podocnemis expansa, número de ninhos marcados, para modelo ARIMA $(2,1,2)$ de 1985 a 2010 com previsão de cinco anos.

As projeções realizadas para todas as séries da produção agropecuária e reprodução dos quelônios, apresentaram na sua maioria um potencial de crescimento no período analisado, com exceção das áreas destinadas a LP (Figura 6).

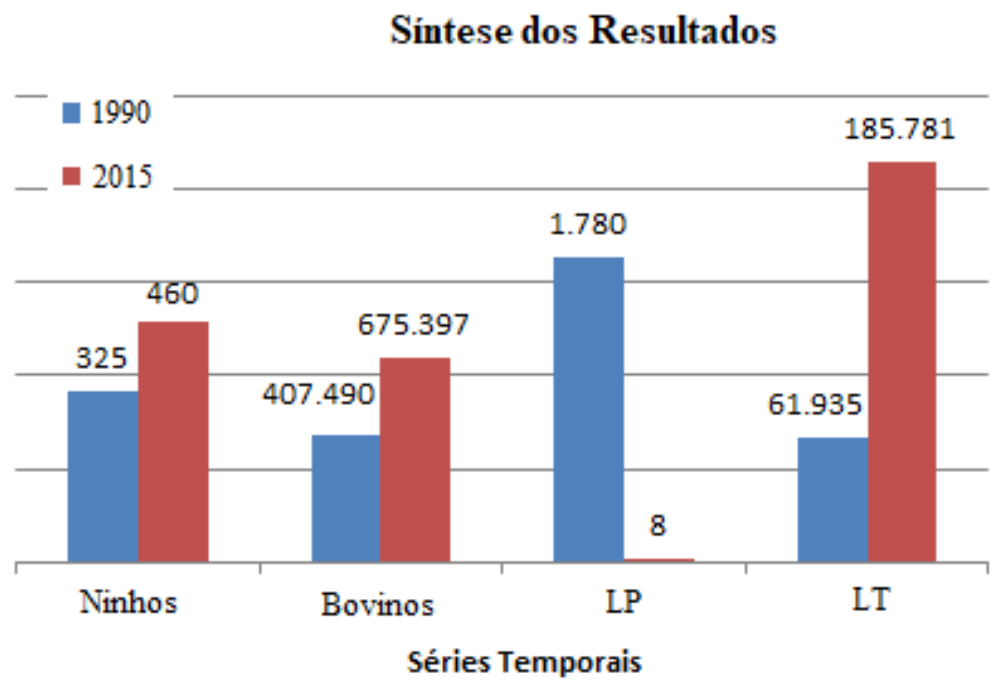

Figura 6. Produção agropecuária e reprodução da Podocnemis expansa. Fonte dos dados: (IBGE/Sidra, 2017; PQA/IBAMA, 2016).

Diversos fatores ambientais podem influenciar na dinâmica reprodutiva da $P$. expansa e consequentemente no tamanho de sua população adulta. Para Alves-Júnior et al. (2012), a temperatura, umidade e precipitação estão diretamente ligados aos aspectos reprodutivos da 
espécie. Ainda citam fatores advindos das ações antrópicas como o desflorestamento e a presença de substâncias químicas na água, advindos da produção agrícola.

Segundo estudos de Packard et al. (1987), os ovos de alguns répteis, apresentaram aspectos diferentes do normal, com manchas escuras quando analisados nos locais próximos a regiões onde as atividades agrícolas eram mais intensas. O uso de agrotóxicos e fertilizantes em regiões com elevada atividade agrícola, pode ser um indicativo da alteração das propriedades da água, que também podem estar interferindo nos aspectos saudáveis dos ovos da $P$. expansa (LOPES, 2016).

Para Frazer (1992), a solução para proteger quelônios do excesso de exploração é minimizar o impacto humano sobre suas populações e principalmente sobre seu hábitat. De La Ossa e Vogt (2011), salienta que os quelônios são suscetíveis à destruição do seu hábitat. Para Mitchell e Klemens (2000), as principais causas do declínio da população ou perda da diversidade de tartarugas estão associadas à perda de hábitat e degradação dos ecossistemas.

Estudos mostram que a $P$. expansa é extremamente sensível às alterações antrópicas, uma vez que é bastante seletiva ao local de desova, preferindo praias altas e grandes (NASCIMENTO, 2002). Nesse sentido as áreas protegidas como o PNA, APA e PEC são extremamente eficazes para a manutenção das populações viáveis de espécies ameaçadas ou espécies potencialmente impactada pela ocupação humana (RODRIGUES et al., 2003; SANCHEZ-AZOFEIFA et al., 2003; VERÍSSIMO et al., 2011). O crescimento da população humana, o aumento das áreas desmatadas, a caça irregular e o aumento das áreas de pastagens para agropecuária ameaçam a sobrevivência de muitas espécies selvagens (SCHNEIDER et al., 2011).

Por outro lado, Simoncini et al. (2016), ao analisar os dados de nidificação do PQA de 1985 a 2008, relata que essa variação no número de animais nidificando está relacionado a variáveis climáticas.

Simoncini et al. (2016), constatou que quanto maior for a precipitação na nascente do rio no mês de maio, maior é a quantidade de fêmeas que desovam nas praias de nidificação. Por outro lado, quanto maior for a quantidade de milímetros precipitados no mês de maio, mais ovos e filhotes serão produzidos nas praias durante a temporada reprodutiva (agosto-outubro).

A influência dessas variáveis na nascente do rio é uma importante ferramenta que permite estimar a quantidade de ninhos nas praias. Mesmo ocorrendo grande variação do número de ninhos no período analisado, no geral, houve um crescimento anual de $1,78 \%$ dos animais em fase de reprodução (Figura 5).

Clerke e Alford (1993), estudando a relação das mudanças climáticas nos répteis, verificaram que as chuvas são um dos fatores mais importantes na sua reprodução. Para Castro e Silva (2005), as chuvas afetam a disponibilidade de presas, e influenciam na taxa de crescimento 
e na energia investida na reprodução (SEIGEL e FITCH, 1985; CAMPOS e MAGNUSSON, 1995; CRUZ et al., 1999).

Para Simoncini et al. (2011), existe uma relação indireta entre as chuvas e a disponibilidade de alimento, o que estaria afetando a incidência reprodutiva de $P$. expansa, e consequentemente seu tamanho populacional. O aumento das chuvas na nascente do rio, antes do período reprodutivo de alguns répteis, poderia trazer benefícios aumentando a disponibilidade de alimentos e possibilitando uma melhor condição física dos animais, possibilitando melhores condições de reprodução na próxima temporada.

\section{CONSIDERAÇÕES FINAIS}

Nota-se que ao longo do período analisado (1990 a 2015), que em 1990, 73,5\% da área era coberta por formações savânicas (domínio de Cerrado), enquanto que em 2015 o quantitativo passou para $62,1 \%$, um decréscimo de $11,4 \%$ nessas áreas. Em relação às formações florestais, em 1990, a região era coberta por cerca de aproximadamente 7\% dessa formação, e 2015 este quantitativo chegou a 5,3\%, houve um decréscimo de cerca de 1,7\% de suas áreas. Em relação ao uso da terra (áreas antrópicas) esta classe que envolve todos os tipos de exploração econômica foi acrescida em mais de $10 \%$.

Avaliando os números da produção agropecuária no período, as principais mudanças de cobertura natural ocorreram com a substituição das áreas de formação savânica, num total de aproximadamente $11 \%$ de conversão. As áreas com formação florestal foram convertidas em aproximadamente $0,8 \%$ para a atividade agrícola. Inversamente à tendência de conversão de áreas nativas para uso antrópico, 47,8\% das áreas de formação savânica mantiveram-se como formação savânica e 4,3\% das áreas e formação florestal mantiveram-se inalteradas.

As áreas antrópicas da região que se destinam a produção agrícola passaram de cerca 63.715 hectares em 1990 para 198.169 hectares em 2015, o que corresponde a um aumento de 134.454 hectares. O percentual dessa área passou de 1,61\% da área total em 1990 para 4,9\% em 2015, o que representa uma taxa de crescimento média de 1,8\% ao ano.

Em relação a população de fêmeas de $P$. expansa em fase de reprodução e sua relação com a produção agropecuária, a análise das séries temporais não permitiu estabelecer um padrão de comportamento entre a quantidade de animais com as das áreas destinadas a LT e LP, assim como, para o aumento da produção de bovinos de corte.

Portanto, na região em análise, a produção agropecuária não pode ser apontada como o principal fator responsável pela variação da quantidade de fêmeas de $P$. expansa em fase de reprodução nas praias do rio Javaés.

Esses resultados dizem respeito a série histórica analisada para produção agropecuária e para nidificação dos quelônios da espécie P. expansa de 1990 a 2015 e 1985 a 2010, 
respectivamente. No entanto, verifica-se um significativo crescimento e avanço da produção agrícola através do uso de terras destinadas a LT e LP próximas as UCs presentes na região, assim como, o crescimento da produção de gado de corte. Mesmo não sendo possível prever o comportamento reprodutivo dos quelônios a partir dos dados da produção agropecuária, é de suma importância a preservação dos locais de reprodução, garantindo assim a sobrevivência da espécie a curto e longo prazo.

Possivelmente, outras ações antrópicas "aparentemente" causadoras de menor impacto ao meio ambiente tenham mais efeitos sobre a população desses animais no que se refere a quantidade de ninhos nas praias do rio Javaés.

A complexidade epistemológica em trabalhos que envolvem estudos comparativos entre informações espaciais e pesquisas socioeconômicas dessa natureza está em tornar compatíveis as informações temporais e conceituais. Aqui, os conceitos envolvidos e os materiais utilizados foram analisados com vistas a minimizar o problema da compatibilidade temporal entre as diferentes fontes de informações.

\section{AGRADECIMENTOS}

À Universidade Federal do Tocantins (UFT), ao Programa de Pós-Graduação em Ciências do Ambiente/UFT, e ao Instituto de Educação Ciência e Tecnologia do Tocantins, Campus Palmas.

\section{REFERÊNCIAS}

ABRAHAM, B.; LEDOLTER, J. Statistical Methods for Forecasting. New York, John Wiley and Sons, 1983. $445 \mathrm{p}$.

ALVES-JÚNIOR, J. R. F.; LUSTOSA, A. P. G.; BOSSO, A. C. S.; BALESTRA, R. A. M.; BASTOS, L. F.; MIRANDA, L. B.; SANTOS, A. L. Q. Reproductive indices in natural nests of giant Amazon river turtles Podocnemis expansa (Schweigger, 1812) (Testudines, Podocnemididae) in the Environmental Protection Area Meanders of the Araguaia river Brazil. Brazilian Journal of Biology, 72(1), 199-203, 2012.

ALHO, C. J. R.; PÁDUA, F. M. Reproductive parameters and nesting behavior of the Amazon turtle Podocnemis expansa (Testudinata: Pelomedusidae) in Brazil. Canadian Journal of Zoology, 60(1), 97-103, 1982.

ARÊDES, A. F.; PEREIRA, M. W. G. Potencialidade da utilização de modelos de Séries temporais na previsão do preço do trigo no estado do Paraná. Revista de Economia Agrícola, 55(1), 63-76, 2008.

AtAíDES, A. G; MALVÁSIO, A.; PARENTE, T.G. Percepções sobre o consumo de quelônios no entorno do Parque Nacional do Araguaia, Tocantins: conhecimentos para conservação. Gaia Scientia, 4(1), 07-20, 2010. 
BOX, G. E. P.; JENKINS, G. M. Time series analysis forecasting and control. San Francisco: Holden Day. Edição revisada. 1976.

BOX, G. E. P.; JENKINS, G. M.; REINSEL, G. C. Time series analysis - forescasting and control, 3aㅡ ed., Prentice Hall, New Jersey. 1994.

BOUR, R. Global diversity of turtles (Chelonni; Reptilia) in freshwater. Hydrobiologia, 595, 593598, 2008.

CAMPOS, Z., MAGNUSSON, W. Relationships between rainfall, nesting habitat and fecundity of Caiman crocodilus yacare in the Pantanal, Brazil. Journal of Tropical Ecology, 11(3), 353-358. 1995.

CLERKE, R. B., ALFORD, R. A. Reproductive biology of four species of tropical Australian Lizards and comments on the factors regulating lizard reproductive cycles. Journal of Herpetology, 27(4), 400-406, 1993.

CRUZ, F. B.; TEISAIRE, E.; NIETO, L.; ROLDÁN, A. Reproductive biology of Teius teyou (Squamata: Teiidae), in the semiarid chaco, Salta, Argentina. Journal of Herpetology, 33(3), 42029, 1999.

CASTRO, M. L.; SILVA, J. A. L. Mathematical modelling of the Ibera Caiman yacaré. Ecological Modelling, 186(1), 99-109, 2005.

DE LA OSSA, J. R. C., VOGT, R. Ecología poblacional de Peltocephalus dumerilianus (Testudines, Podocnemididae) en dos tributarios del río Negro, Amazonas, Brasil. Interciencia, 36(1), 53-58, 2011.

DICKEY, P. A.; FULLER, W. A. Distribution of Estimators for Autoregressive Time Series With a Unit Root. Journal of the American Statistical Association, 74(366), 427-431, 1979.

FAVA. V. L. Manual de econometría. In: WERNER, L.; RIBEIRO, J. L. D. Previsão de Demanda: Uma Aplicação dos Modelos de Box-Jenkins na Área de Assistência Técnica de Computadores Pessoais. 2000.

FELFILI, J. M.; CARVALHO, F. A.; HAIDAR, R. F. Manual para o monitoramento de parcelas permanentes nos biomas Cerrado e Pantanal. Universidade de Brasília, Brasília, Distrito Federal. 55 p. 2005.

FISCHER, S. Séries univariantes de tempo: metodologia de Box e Jenkins. $1982186 \mathrm{f}$. Dissertação (Mestrado em Economia) - Universidade Federal do Rio Grande do Sul, Porto Alegre, 1982.

FRAZER, N. B. Sea Turtle Conservation and Halfway Technology. Conservation Biology, 6(2), 179-184, 1992.

FREUND, J. E.; SIMON, G. A. Estatística Aplicada, 9ae edição, Editora Bookman. 2000.

GRANGER, C. W. J.; NEWBOLD, P. Forecasting Economic Time Series. London, Academic Press, 1986. 211 p.

IBAMA - Instituto Brasileiro do Meio Ambiente e dos Recursos Naturais Renováveis. Área de Proteção Ambiental - APA - Meandros do rio Araguaia: Relatório. Goiânia, GO. 55 p. 2004.

IBAMA - Instituto Brasileiro do Meio Ambiente e dos Recursos Naturais Renováveis. Programa Quelônios da Amazônia - (IBAMA/PQA). Disponível em: http://www.ibama.gov.br/faunasilvestre/programa-quelonios-da-amazonia. Acesso em: 30 nov. 2016. 
IBGE - Instituto Brasileiro de Geografia e Estatística. Sistema de Contas Regionais. Disponível em: http://www.ibge.gov.br/. 2007. Acesso em: 19 jun. 2016.

IBGE - Instituto Brasileiro de Geografia e Estatística. Produção Agrícola Municipal 1990 - 2015. Sistema IBGE de Recuperação Automática - SIDRA. Disponível em http://www.sidra.ibge.gov.br/bda/. Acesso em: 19 jun. 2017.

IUCN - INTERNATIONAL UNION FOR CONSERVATION OF NATURE. The IUCN Red List of Threatened Species. Cambridge, 2016. Disponível em: http://www.iucnredlist.org/. Acesso em: 20 mai. 2016.

ICMBIO. Diagnóstico da Fauna - Avaliação científica do risco de extinção da fauna brasileira. Coordenação de Avaliação do Estado de Conservação da Biodiversidade Coabio/CGESP/ICMBio. Brasília: ICMBio. 2014. 40 p. (Biodiversidade Brasileira).

MAKRIDAKIS, S.; WHEELWRIGHT, S.; HYNDMAN, R. J. Forecasting methods and applications. 3. ed. New York: John Wiley and Sons, 1998.

LOPES, T. K. M. Estudo da Característica Estrutural das Praias de Nidificação de Podocnemis Expansa e dos Impactos Potenciais Decorrentes da Atividade Antrópica dm Áreas Ribeirinhas da Bacia do Araguaia - TO. 2016. 88 f. Dissertação (Mestrado em Ciências do Ambiente) Universidade Federal do Tocantins, Palmas, 2016.

MARCO, A.; PÉREZ, E. A.; ARGÜELlO, C. M.; MARTINS, S.; ARAUJO, S.; JURADO, L. F. L. The international importance of the archipelago of Cape Verde for marine turtles, in particular the loggerhead turtle Caretta caretta. Zoologia Caboverdiana, 2(1), 1-11, 2011.

MARCOVALDI, M. A.; LOPEZ, G. L.; SANTOS, A. J. B.; BELLINI, C.; SANTOS, A. S.; LOPEZ, M. Avaliação do Estado de Conservação da Tartaruga Marinha Eretmochelys imbricata (Linnaeus, 1766) no Brasil. Biodiversidade Brasileira, 1, 20-27, 2011.

MARCOVALDI, M. A.; MARCOVALDI, G. G. Projeto Tamar: área de desova, ocorrência e distribuição das espécies, época de reprodução, comportamento de postura e técnicas de conservação das tartarugas marinhas no Brasil. Brasília, MA-IBDF. 46 p. 1985.

MARQUÉZ, C. História natural y dimorfismo sexual de la tortuga Kinosternon scorpioides en Palo Verde, Costa Rica. Revista de Ecología Latino-Americana, 2(1), 37- 44, 1995.

MITCHELL C. J.; KLEMENS, C. M. Primary and secubdary effects of habitat alteration. Klement CM. (ed). Turtle Conservation. Smithson. Inst. Press; 2000.

MMA - Ministério do Meio Ambiente. Espécies Brasileiras Ameaçadas de Extinção, Sobreexplotadas ou Ameaçadas de Sobre exploração. 2016. http://www.mma.gov.br/biodiversidade/especies-ameacadas-de-extincao

MMA - Ministério do Meio Ambiente. Plano de Manejo do Parque Nacional do Araguaia - TO. Brasília: MMA. 429 p. 2001.

MORETTIN, P. A.; TOLOI, C. M. C. Previsão de séries temporais. São Paulo: Atual, 1985. 435 p.

MORETTIN, P. A.; TOLOI, C. Análise de séries temporais. Blucher, 2006.

NASCIMENTO, S. P. Observações sobre o comportamento de nidificação de três espécies de Podocnemis Wagler (Testudinata, Pelomedusidae) no Baixo Rio Branco, Roraima, Brasil. Revista Brasileira de Zoologia, 19(1), 201-204, 2002. 
PÁDUA, L. F. M. Biologia da reprodução, conservação e manejo da tartaruga-da-amazônia Podocnemis expansa (Testudines, Pelomedusidae) na Reserva Biológica do Rio Trombetas, Pará. 1981. 133 f. Dissertação (Mestrado) - Universidade de Brasília, Brasília, 1981.

PACKARD, G. C.; PACKARD, M. J.; MILLER, K.; BOARDMAN, T. J. Influence of moisture, temperature, and substrate on snapping turtle eggs and embryos. Ecology, 68(4): 983-993. 1987.

PELLEGRINI, F. R.; FOGLIATTO, F. S. Passos para Implantação de Sistemas de Previsão de demanda - Técnicas e Estudo de Casos. 2000.

REBÊLO, G. H.; PEZZUTI, J. C. B. Percepções sobre o consumo de quelônios na Amazônia: Sustentabilidade e alternativas ao manejo atual. Ambiente e Sociedade, 6/7, 85-104, 2000.

RODRIGUES, A. S. L.; ANDELMAN, S. J.; BAKARR, M. I., Global gap analysis: towards a representative network of protected areas. Advances in applied biodiversity science, 5, (ed. by P.J. Benson and N. Lindeman), Washington, DC: Conservation International. p. 1-98. 2003.

SANCHEZ-AZOFEIFA, G. A.; DAILY, G.; PFAFF, A.; BUSCH, C. Integrity and isolation of Costa Rica's national parks and biological reserves: examining the dynamics of land-cover change. Biological Conservation, 109(1), 123-135, 2003.

SCHNEIDER, L.; FERRARA, C. R.; VOGT, R. C.; BURGER, J. History of Turtle Exploitation and Management Techniques to Conserve Turtles in the rio Negro Basin of the Brazilian Amazon. Chelonian Conservation and Biology, 10(1), 149-157, 2011.

SEIGEL RA, FITCH HS. Annual variation in reproduction in snakes in a fluctuating environment. Journal Animal Ecology, 54(2), 497-505, 1985.

SIMONCINI, M.; PIÑA, C.I.; CRUZ, F.B.; LARRIERA, A. Climatic effects on the reproductive biology of Caiman latirostris (Crocodylia: Alligatoridae). Amphibia Reptilia, 32(3), 305-314, 2011.

SIMONCINI, M., THIAGO C.G. P., KENNEDY M. M., GUTH B. F., ERICH C., RAFAEL A. M. B., VERA L. F. L., ADRIANA M. Avaliação da influência dos fatores ambientais sazonais na reprodução da tartaruga-da-amazônia (Podocnemis expansa) em Tocantins. Universidade Federal do Tocantins. Palmas. 2016.

SMITH, N. J. H. Quelônios aquáticos da Amazônia: um recurso ameaçado. Acta Amazônica, Manaus, 9(1), 87-97, 1979.

SOINI, P. Un manual para el manejo de quelonios acuáticos en la amazonia peruana (Charapa, Taricaya y $\quad$ Cupiso). $1999 . \quad$ Disponível http://repositorio.iiap.org.pe/bitstream/IIAP/121/2/Soini_Libro_1999.pdf. Acesso em: 15 maio 2017.

SPOTILA, J. R. Sea Turtles: a complete guide to their biology, behavior, and conservation. Baltimore, Johns Hopkins University Press, Oakwood Arts. 2004. 240 p.

VERÍSsIMO, A.; ROLLA, A.; MAIOR, A. P. C. S.; MONTEIRO, A.; BRITO, B. SOUZA JR, C.; AUGUSTO, C. C.; CARDOSO, D.; CONRADO, D.; ARAUJO, E.; RICARDO, F.; RIBEIRO, J.; LIMA, L. M.; RIBEIRO, M. B.; VEDOVETO, M.; MESQUITA, M.; BARRETO, P. G.; SALOMÃO, R.; FUTADA, S. M. Áreas Protegidas na Amazônia brasileira: avanços e desafios. Belém: Imazon Instituto Socioambiental, 2011. 90 p.

VOGT, R. C. Tartarugas da Amazônia. Lima, Peru, 2008. 104 p.

WHEELWRIGHT, N. T. Fruit size, gape width, and the diets of fruit-eating birds. Ecology, 66(3), 808-818, 1985. 
WERNER, L.; RIBEIRO, J. L. D. Previsão de Demanda: Uma Aplicação dos Modelos de BoxJenkins na Área de Assistência Técnica de Computadores Pessoais. Gestão e Produção, 10(1), 47-67 2003.

Revista Ouricuri, Juazeiro, Bahia, v.8, n.2. p.026-048. jul./dez., 2018.

http://www.revistas.uneb.br/index.php/ouricuri | ISSN 2317-0131 